\title{
Nursing student engagement: Researching the journey and its potential impact on transitions to practice
}

\author{
Kathleen Hudson*, Zhaomin He and Rebecca Carrasco \\ University of Texas at Tyler, Longview University Center, USA
}

\begin{abstract}
Nursing student engagement fundamentally contributes toward quality student advancement and future quality nursing care. This research evaluates students' perception of their nursing education and their commitment toward practice. The findings provide a background for the development of solid nursing practices with high scores of engagement, in both class and clinicals. The scores for stress management and coping were the lowest and further point to a greater learning need in this area. Currently doing very well with the learning content, they simultaneously are suffering with challenging emotional responses to this complex education. Stress management, coping, and resiliency sessions are now acutely highlighted as extremely important and necessary (for high quality nursing students'long-term emotional and cognitive wellness). Students can be functioning at higher emotional levels longer with this supportive reinforcement. When this emotion protection is developed, and implemented, students will provide a stronger and more resilient future nursing workforce.
\end{abstract}

\section{Introduction}

Engaged with their learning, excited and motivated, student nurses are enhancing their profession. These students can complement practice and provide new ideas and better ways of performing practice. Student nurses' sense of engagement is extremely high. While experiencing high engagement, the complexity of their role as they experience it and are continually learning, can be overwhelming. These stressful and significant pressures during their nursing education experience include learning complex and critical coursework, various financial issues and responsibilities, social needs and desires of being in an academic setting, spiritual needs, and a diverse range of personal stressors. The specific nursing clinical experiences include exposure to patients within 'stressful, intense, diverse, and impactful' illnesses, learning the intricate details of appropriate clinical care provision. And simultaneously, they must complete rigorous academic activities to gain appropriate knowledge to function competently within various clinical specialties.

As generalists, nurses must be knowledgeable in many areas including:

- Different practice environments,

- Different cultures within the main culture,

- Numerous and diverse medications,

- Usual routines and daily performance expectations,

- Organizational policy and procedures, and

- Nursing's applicable 'best practices' for their specific work settings.

Student nurses experience a dedication and commitment to learning nursing, with all its' complexities, as they prepare for their future clinical roles.

There are various ways to define Nursing Student Engagement (NSE). For example, engagement in particular, involves therapeutic engagement consisting of empathy, active listening and collaboration [1]. Three levels of student engagement are described: engagement between the student and teacher, the student connection with their learning, and the student linking with their learning environment [2]. Our nursing students level of NSE was analyzed at our university settings to review the students' educational and clinical 'preparedness' perceptions and monitor their evolving perspective of their engaged readiness for complex clinical practice. Students at Level two (halfway through the program, L2) and level four (just before graduating, L4) were investigated. This study results found consistent finding with previous research [3] representing students' having intense engagement with their studies, their educators and fellow students. However, they also revealed ongoing personal weaknesses related to 'stresses' and unhealthy 'coping'. Hudson [4] developed a working model for coping that includes descriptors helping to reveal the complexity of coping for nurses. From the findings of this previous research, we supported and complimented our efforts toward maintaining high nursing student engagement and planning toward developing healthier coping strategies. This study validates the need for solidifying strong building blocks toward enhancing positive coping skills and quality resilience building.

\section{Background}

Engagement (NSE) comprises three main components: behavioral, emotional, and cognitive engagement [5]. These three engagement aspects include attentiveness, active participation, commitment,

${ }^{\star}$ Correspondence to: Kathleen Hudson, University of Texas at Tyler, Longview University Center, USA, Tel: 903-240-7040, E-mail: KHudson@uttyler.edu

Key words: nursing student engagement, coping, resiliency, stress management, quality, education needs

Received: November 22, 2018; Accepted: January 16, 2019; Published: January 25,2019 
intuition, collaboration, teamwork, creativity, and integrative thinking [3] . The aspects of 'engaged relationships' between the nursing learning institution and key relevant parties including: the institution, fellow students, and teachers are identified below on the Nursing Student Engagement Network Model (Figure 1).

Strong feelings of engagement are critical for ethical and participative caring for others. Highly engaged nurses are more likely to be more committed to quality decision making when solving their patients' problems. Engaged nurses will work through tough problems with commitment toward the patients' best interests, not necessarily their own ease or convenience. This is high quality health care, focused on the patients' sense of well-being. Even 'troublesome' patients can be cared for with quality by engaged nurses. These engaged nurses are aware of the issues comprising the patient's presentation and will overlook the negativity and work toward moving them forward toward better health. Engaged nurses cope positively with challenges and have likely developed more resilience than their colleagues have. The end goal of developing strong resilience is a long-term goal for nursing students: Students desire to become great caring practitioners, providing the best care possible. Resiliency can help them maintain this goal as they work to achieve an ongoing high standard of clinical practice.

In developing this sense of commitment within their profession, students appreciate positive nurturing and reinforcement from others during their nursing education. The nurse educators (instructors), clinical nursing mentors, and peers become the 'learning stick' in which to measure care education, care provision, and 'nursing quality'. The nurse educators must readily discuss and review patient issues. They must describe what is yet 'to be experienced' by the students. The educators also need to role model quality caring and the desired decision-making behaviors. Thus, critical thinking should evolve mirroring what they have learned and seen. Thereafter, when completed well, a student's decision-making, reflection and consideration, becomes their experiential knowledge and thereafter, benefit future positive decision-making experiences.

On the other hand, students exposed to negativity and disengagement from some nurses or others in a clinical setting are also

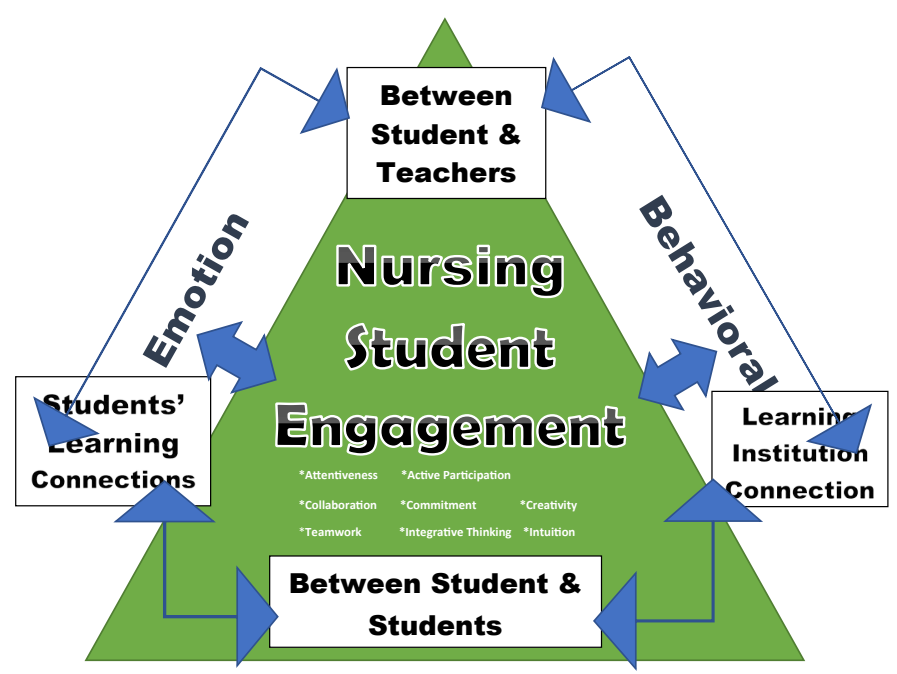

\section{Cognitive}

Figure 1. Nursing Student Engagement Multidimensional Network (Hudson \& Carrasco, 2015, expanded by Hudson, 2018) important topics to discuss. They can be exposed to these situations in various clinical settings and personally decide the specific type of nurse they would like to be (positive or negative) and professionally present this role modeling to others. Specific discussions of poor clinical behaviors will expose the student to less than optimal care, risk of care errors, patient mistreatment, and the consequential negative impact on the care environment. Consistently, this situation is followed by students' comments of, 'I never want to be like that'. This is important for their 'professional framing' of the type of nurse they will become.

\section{Engagement's Current Colors}

Crookes, Crookes, and Walsh [6] studied engaging techniques for nursing students and sometimes found a 'disconnected tension' between theory and practice. This is due to students being more 'relevancy orientated' and desiring their learning environments to promote 'practical use' of what they are studying. Most nursing schools today provide active learning situations in virtual simulation labs and case review scenarios where students work through solving actual patients' problems. These sessions readily stimulate engagement for students when the sessions are conducted well.

As lectures are occurring, excellent educators present contemporary problems, which will then be applied to related theories as presented. This is 'real application' learning, readily applicable to the clinical settings. This style of active learning is successful at maintaining student engagement and enhances content retention. The advantage of this discussion format is that it ties the theory into the case scenarios that can provide some transfer of this knowledge to comparable situations. For example, when teaching and discussing dying patients, the theoretical pathway of dying stages and death can be presented. This assists to provide examples to subsequently use for specific care decision-making efforts; which are appropriate for that specific dying phase. This problem-based learning enhances student reflection and decision-making and provides a solid background for expansion of students' experiential learning.

This learning development provides the background for better stress management and coping. The scenarios are 'life-like' and coping strategies can be reasoned through, as students work through various options, and potential outcomes subsequently analyzed. These positive coping strategies are critical for attainment of positive outcomes. Understandably, these positive outcomes apply to patients' as well as nursing students' coping strategies as the problems are untangled.

Today, important learning tactics include activities that increase students' interactions and cooperation with other professionals. Some on-line groups and in-person activities can assist with creating situations where students are familiar and comfortable with their clinical co-workers such as doctors, physical therapists, nurse aides, and secretaries, and other health care workers. Role-playing with communication of various issues plays an important component in the exercises geared toward providing excellent care. When any of these 'roles' are not fully engaged, the care becomes compromised and 'at risk' for errors. Nursing students and others can identify these risk situations and can make adjustments to avoid any errors. One example already standardized for clinical practice would be restating communication orders to provide for the double-checking of care provision. This is critical for patient safety, as patients are at risk of harm and delays in care when errors occur. These are important aspects for students to come to terms with so they can prevent issues from arising by utilizing proven prevention strategies. Being a safe practitioner is vital for feelings of engagement with nursing practice. 
Many innovations for bedside care are transitioning care delivery for nurses. One such innovation is handheld devices to enhance the speed and accuracy of care delivery. Nurses are using frequently improved handheld devices to communicate, record, deliver medications, monitor, investigate, clarify, alert, and alarm as needed. Students are learning about tools that will soon be part of their standard clinical practice. Handheld clinical data devices can communicate data quickly and accurately, much better than any person's memory. Utilizing these devices with patients can encourage patients to also engage with routine accessing of important on-line information for their care. This type of on-line data driven care is very appealing to today's students. They are very engaged in learning about quick and accurate decisionmaking with their patients due to the accuracy and quick access to this important information. Having reliable tools to communicate critical information also strengthens engagement within a busy clinical environment.

Academic education is now on-line for many programs. This learning involves more personal enjoyment and engagement with selfpaced learning activities. This assists with creating a positive learning experience. When this style of learning is used as a group activity, it aligns with how many of today's students desire to learn. Any course without direct patient care, such as nursing concepts, is now on line at our institution. Encouraging and maintaining engagement is critical as students can feel detached for their fellow students and teachers when their courses are on line.

However, an important part of nursing care is the interpersonal interaction between patients and nurses. How are nursing students learning to refine their social interactions - to accurately communicate, interpret, and make relevant decisions when they provide care - if the core of their interactions are all on-line? How can instructors quickly pick up if students are feeling less engaged with the course(s)? Using frequent quality communication and visualizing others on screen during discussions can minimize feelings of detachment while still maintaining physical distances from the education source.

When consolidating clinical training, nothing is better than direct discussion of patients with a stimulating post-conference discussion. This provides actual experiential learning, which can generate more understanding and context for student nurses. This direct clinical hands-on and post-clinical discussion is still a critical part of baccalaureate nursing training at our institution. The establishment of 'best practice' educational standards between on-line, simulation, hybrid, and supervised direct patient care learning is yet to universally determined.

In the meta-construct of engagement, the three components: emotion, cognition, and behavior have elements enacting on them such as: active participation, collaboration, and teamwork among others [3]. "Good teamwork and coordination" skills were found to be necessary elements for beginning nurses to be successful in the first year of practice [7]. Some universities are starting to use on-line interprofessional education (IPE) to sharpen the necessary clinical skills need for today's work environments. They are using a virtual IPE curriculum to enhance the cooperation, collaboration and results of group decision making within the healthcare context. This higher level of interdepartmental communication is long overdue. Determining the best and most effective methods to teach interdisciplinary groups is yet to be established. However, bridges 'are being formed' to improve engagement, decrease incivility, and provide transparent communication between professionals, in both practice and academia [8].
In the postmodern society where information is best understood in context, students learn through a diverse range of engaging activities, such as gaming. Davidson and Candy [9] used game-based learning in an online evidenced-based practice course achieving improved academic outcomes and greater student satisfaction in the course.

Djukic et al. [10] exemplify another form of engaged learning:

'The Institute of Medicine (IOM) has identified interprofessional education as a key innovation for achieving the triple aim of better care, better outcomes, and reduced health care costs.'

Using a multidisciplinary virtual IPE curriculum to enhance the cooperation, collaboration and results of group decision-making is important within the healthcare context. For nursing, the outcomes resulted in significantly higher working knowledge of teamwork, communication and conflict resolution, and interprofessional planning. These results will enhance engagement for nursing students, and they will bring these collaborative skills to the bedside 'worksite'.

As a priority, clinical settings are selected which can best meet the students' current learning needs. Students are expose to various preceptors and charge nurses, all playing a part in the role modeling which the student is 'observing'. Even when the situation is not desirable, this is a great learning opportunity to demonstrate 'what is not' as effective as compared to 'what is'. These scenarios discussed as an option during clinical debriefings readily become great learning opportunities. Debriefing to consolidate the 'critiqued care and reflection-based knowledge learning' is highly desirable for students. This is how strong educators can provide valuable student feedback, correct discrepancies as needed, and direct the learning of complex care situations.

Our study, which investigated nursing students across our three campus locations, researched 'nursing student engagement' at our university. The research questions were:

1). What are the current levels of nursing student engagement at our university, at each of the three campuses? And then compared?

2). From the 'nursing student engagement perceptions' information collected, what are the identified areas of difficulties/challenges, and therefore, areas for improvement?

\section{Methods}

\section{Sample and Procedure}

In relation to ethical considerations, the university's Institutional Review Board and the nursing department's leadership approved this study. The study involved administering a self-administered, anonymous survey, the Student Engagement Questionnaire [11], which assisted to determine the students' perception of their level of student engagement.

Data was to be collected during class time from the three campus nursing education sites. There were no exclusions for this survey. Each site included two levels of nursing education from an eastern Texas University, the second level (L2) and the fourth level (L4). All students registered and participating in the class were eligible. Students were all provided an explanation of the study's purpose. Then according to the ethical considerations, students were requested to complete the questionnaire voluntarily and anonymously. All students who were in class on the day of the survey's distribution were very interested in filing out the survey and writing about their school experience; each 
student filled out a consent form to participate in this study, and then independently completed the instrument.

In conclusion, students were provided the explanation in consideration of ethical rights, consent forms completed, and the questionnaire was distributed (no student identifiers were on the questionnaires), and students returned the questionnaires into a folder after they were completed.

\section{Instrument}

The Student Engagement Questionnaire [11,12] was used to measure student engagement on a 5-point Liker scale. The questionnaire consists of 35 items and five subscales:

(1) intellectual (12 items),

(2) working together (4 items),

(3) teaching (11 items),

(4) teacher-student relationship (4 items), and

(5) student-students relationship (4 items).

The rating scale of 1-5 represented: (5) strongly agree, (4) agree, (3) only to be used if a definitive answer is not possible, (2) disagree, and (1) strongly disagree.

The five subscales each cover multiple aspects of engagement. The specific areas assessed were the students' perception of their critical thinking, creative thinking, self-management, adaptability, problem solving, communication, interpersonal and group work skills, active learning, teaching for understanding, feedback to assist learning, assessment, relationship with teachers and students, workload, cooperative learning, and cohesion of the curriculum [3].

The overall reliability based on the sample in this study was .94 and the internal consistency coefficients for the five subscales were all above .80 , specifically .89 (intellectual), .83 (working together), .89 (teaching), .90 (teacher-student relationship), and.82 (student-students relationship) respectively.

\section{Analysis}

First, the means were calculated for the subscales, as well as, the overall student engagement mean to be used in the subsequent statistical analyses. Descriptive information was obtained; and normality was examined for the distribution of these mean scores. Due to absence of normality, independent samples Mann-Whitney's U (for Level) and Kruskal-Wallis tests (for Campus) were used to examine the differences in student engagement between the two levels and across the three campus sites.

\section{Results}

A total of 251 undergraduate nursing students responded from three undergraduate nursing sites. Four student responses were excluded due to incomplete surveys. Out of the resulting 247 students, $67.2 \%$ were from the main campus, $15.4 \%$ and $17.4 \%$ from the other two campuses respectively. Over half of the participants were level two students $(56.3 \%)$, and the remainder were level four graduating students.

The descriptive information described the students as rating high on overall engagement and all the five subscales (see Descriptives of Student Engagement and the Five Subscale Mean Scores, Table 1). The same pattern was also found in their average rating scores by level and campus.

Results of the distribution statistics and normality tests confirmed this pattern indicating presence of non-normality. Given the violation of the normality assumption, parametric tests were used to investigate the differences between levels and across campuses in student engagement. Results of the non-parametric tests showed significant differences in student overall Engagement between Levels 2 and 4 ( $p=$ .013) but not across campus sites.

Significant differences were also individually analyzed between the various levels, results found were:

- Intellectual between levels ( $p=.005)$ but not across campus sites

- Working Together between levels $(p=.014)$ and across campuses ( $p$ $=1.026)$ Teaching between levels $(p=.023)$ but not across campuses

- Teacher-Student Relationship across campuses ( $p=.017)$ but not between levels Student-Students relationship across campuses ( $p=$ .015) but not between levels

\section{Qualitative results of this survey included}

In relation to clinical times and clinical experiences, students felt these were the best aspects of the program. They also appreciate SIMS lab and skills lab sessions. Students reported high levels of 'teamwork and collaboration".

An overwhelming positive response to their teachers was reported, such as, "willingness, make effort to help, wanting you to learn, hands on, willing to help to better understand students, helpful, open door policy, personable, communication, and truly want students to excel".

Areas the nursing program needed to improve were identifies as: "workload, more time to complete skills, making arrangements to complete skills, and advising".

Some students (8) reported they did have personal "stress and anxiety experiences"; a few had never had experiences with being part of the caregivers for people with mental illnesses before their level 2 semester (9). The perception of stress varies from one student to another. Stress did appear to be a regularly reported concern of students. They reported needing: "help with test anxiety", better 'time management', help with the 'interview process', "never had any experience with mental illnesses", "getting saturated", "more time

Table 1. Descriptive of Student Engagement and the Five Subscale Mean Scores, M (SD)

\begin{tabular}{|c|c|c|c|c|c|c|c|}
\hline & & Engagement & Intellectual & Working Together & Teaching & $\begin{array}{l}\text { Teacher-Student } \\
\text { Relationship }\end{array}$ & $\begin{array}{c}\text { Student-Students } \\
\text { Relationship }\end{array}$ \\
\hline \multicolumn{2}{|l|}{ Overall (SD) } & $4.20(.48)$ & $4.39(.46)$ & $4.31(.57)$ & $3.99(.64)$ & $4.07(.85)$ & $4.12(.72)$ \\
\hline \multirow{2}{*}{ Level (SD) } & 2 & $4.12(.04))$ & $4.32(.04)$ & $4.26(.05)$ & $3.92(.06)$ & $3.99(.08)$ & $4.09(.06)$ \\
\hline & 4 & $4.29(.04)$ & $4.49(.04)$ & $4.43(.05)$ & $4.12(.05)$ & $4.18(.07)$ & $4.17(.07)$ \\
\hline \multirow{3}{*}{ Campus (SD) } & 1 & $4.18(.04)$ & $4.39(.04)$ & $4.37(.04)$ & $3.94(.05)$ & $4.03(.07)$ & $4.12(.05)$ \\
\hline & 2 & $4.35(.06)$ & $4.45(.07)$ & $4.39(.7)$ & $4.20(.07)$ & $4.40(.08)$ & $4.41(.08)$ \\
\hline & 3 & $4.15(.07)$ & $4.37(.06)$ & $4.12(.9)$ & $4.09(.08)$ & $3.96(.13)$ & $3.88(.15)$ \\
\hline
\end{tabular}


for skills", "my stress level has increased since before this semester", "I have increased anxiety", and "I did not realize how susceptible to anxiety and stress I am".

Some reported good experiences such as, "being able to do clinicals in different aspects of healthcare", "focused on critical thinking with encouraging holistic perspective and seeing the whole process", "developing communication skills", "liked the simulation exercises", and enjoyed "the friendships gained".

The results demonstrated strong perceptions of NSE during their attendance at the university nursing program. One area was a significant concern for students. This area was generally related to stress and coping management.

\section{Discussion}

What is the students' nursing journey? The journey for nursing students is through the learning process at university. This journey involves working towards graduating, and is laborious and demanding. The outcome is rewarding and fulfilling. Students look to the future with anticipation, uncertainty, and hope toward a fulfilling career within their new profession of nursing.

"How do you care for someone else when you can't or don't care for yourself?" Certainly, a student's stress level can damage their learning experiences and their ability to concentrate and cope in a positive fashion. Stress management is a focus area for students due to these reports. We are developing coping and resiliency training sessions to be included within the nursing program.

What is the potential impact for transitions to practice? We would all agree that the students are not completely ready for practice. Students always head to their new practice arenas without a full complement of 'practiced' skills. There are 'always holes' in their 'comprehensive training' for numerous reasons. This is due to the numerous and complexity of actual nursing roles. In addition, the diverse clinical settings are each somewhat unique in layout, culture, training, and support systems. These units vary from hospital to hospital (hospitals are where most nurses start their careers). This situation causes stress, anxiety, a lack in confidence, and primarily, a risk for patients. Finding better ways for students to fully prepare better and feel better about their preparedness is daunting. The first year of clinical practice should support the gradual development of the necessary skills needed for working within the specific clinical environment to which the new nurse starts working.

Having engaged new nurses interested in doing the 'right' thing, and 'communicating well' -to the best of their abilities - is important. With proper support and caring mentors, these new nurses will bloom into well-rounded clinical experts, grounded in their sense of commitment for their patients. The patient outcomes will always be better with 'engaged' nursing professionals making shared decisions.

The diversity in students' nursing preparation is important. Their science programs of 'systems-focused, context-driven approaches' assists students to better gain applicable applications of their knowledge to the practice situations [13]. Making sure the education the nursing students are receiving has the depth to allow timely and accurate critical decision-making for actual clinical practice is fundamental. This appropriate of both breadth and depth of education will provide a safe practitioner baseline for practice. It will also provide a workable learning opportunity to keep new engaged nurses stimulated 'to really learn' more each day, and not just go through the regular staff's motions of providing their usual standard of care. Engaged students look for improving patients' clinical status, enhancing the patients' personal care and wellbeing, decrease their length of stay, and certainly keeping them informed during their stay. New nurses strive for 'true nursing engagement and committed caring for their patients' as the patients proceed through their healing processes.

This evaluation of students' sense of engagement provides a good picture of how they perceive their education and their clinical environments in which they are currently functioning. Our findings highlight the need for development of well-rounded stress management, positive coping strategies, and discussions on how to develop improved resiliency. They are interested in developing positive coping and resiliency skills, toward their self-improvement, and therefore, enhancing their patient care delivery.

\section{Conclusion}

Nursing student engagement is a critical component of the nursing students' learning experience. Our abilities to support students through their studies, providing courses, direction and correction, and communication and documentation skills, and the many other features of university education needs to be constantly analyzed. What this research has specifically revealed is the missing identified necessity of students feeling strongly about their ability to cope positively. Stress management, time management, interpersonal management, conflict management, and many other psychiatric skills for the nursing students need further course integration, practice, and refinement. The overall goal of nursing students being successful in their future practice relies on the refinement of these skills so the students will be more balanced, receptive to addressing the various conflicts that arise in practice, and better 'self-carers'. The goals of providing caring, healthy, adaptable, and competent quality nurses for our communities relies on their abilities to be engaged with their decision making and their ability to cope with what they face - each day.

\section{References}

1. Bright FAS, Kayes NM, Worrall L, McPherson KM (2014) A Conceptual Review of Engagement in Healthcare and Rehabilitation. Disabil Rehabil 37: 643-654. [Crossref]

2. Bowcock R, Peters K (2016) Discussion Paper: Conceptual Comparison of Student and Therapeutic Engagement. Nurse Educ Pract 17: 188-191. [Crossref]

3. Hudson K, Carrasco R (2015) Researching nursing students' engagement: Successful findings for nursing. Int $J$ Nurs Clin Pract 2: 1-5.

4. Hudson K (2016) Coping complexity model: Coping stressors, coping influencing factors, and coping responses. Psychology 7: 300-309.

5. Fredricks J A, Blumenfield PC, Paris AH (2004) School engagement: Potential of the concept, state of the evidence. Review of Educational Research 74: 59-109.

6. Crookes K, Crookes PA, Walsh K (2013) Meaningful and engaging teaching techniques for students: A literature review. Nurse Educ Pract 13: 239-243. [Crossref]

7. Hudson K, Carrasco R (2017) Nursing student engagement: Taking a closer look. Open Journal of Nursing 7: 193-20.

8. McSherry R, Cottis K, Rapson T, Stringer M (2015) Embracing external scrutiny to build bridges and genuine partnerships between education and practice. Nurse Educ Pract 15: 149-154. [Crossref]

9. Davidson SJ, Candy L (2016) Teaching EBP using game-based learning: Improving the student experience. Worldviews Evid Based Nurs 13: 285-293. [Crossref]

10. Djukic M, Adams J, FulmerT, Szyld D, Lee S, (2015) E-learning with virtual teammates: A novel approach to interprofessional education. J Interprof Care 29: 476482. [Crossref]

11. Kempler D (2005) Best practice in outcomes-based teaching and learning at the Chinese University of Hong Kong. McNaught, C., edn, Centre for Learning enhancement and Research. 
Hudson K (2019) Nursing student engagement: Researching the journey and its potential impact on transitions to practice

12. D’Souza MS, Venkatesaperumai R, Radhakrishnan J, Balachandran S (2013) Engagement in clinical learning environment among nursing students: Role of nurse educators. Open Journal of Nursing 3: 25-32.
13. Ralph N, Birks M, Cant R, Tie C, Hillman E (2017) How should science be taught to nurses: Preferences of registered nurses and science teaching academics. Collegian 24 $585-591$.

Copyright: (C2019 Hudson K. This is an open-access article distributed under the terms of the Creative Commons Attribution License, which permits unrestricted use, distribution, and reproduction in any medium, provided the original author and source are credited. 